\title{
Orbifold SUSY GUT from the Heterotic String
}

\author{
Bumseok Kyae \\ Department of Physics and Astronomy and Center for Theoretical Physics, \\ Seoul National University, Seoul 151-747, Korea
}

\begin{abstract}
From the string partition function, we discuss the mass-shell and GSO projection conditions valid for KaluzaKlein (KK) as well as massless states in the heterotic string theory compactified on a nonprime orbifold. Using the obtained conditions we construct a 4D string standard model, which is embedded in a 6D SUSY GUT by including KK states above the compactification scale. We discuss the stringy threshold corrections to gauge couplings, including the Wilson line effects.
\end{abstract}

Keywords: nonprime orbifold, SUSY GUT, heterotic string

PACS: $11.25 . \mathrm{Mj}, 11.25 . \mathrm{Wx}, 12.10 . \mathrm{Kt}$

Introduction. An indirect but the most convincing evidence for the existence of the supersymmetric grand unified theory (SUSY GUT) would be found from the notice that the three gauge couplings in the minimal supersymmetric standard model (MSSM), $\left\{g_{3}, g_{2}, \sqrt{5 / 3} g_{Y}\right\}$ are unified at the $10^{16} \mathrm{GeV}$ scale. Here the non-trivial normalization factor " $\sqrt{5 / 3}$ " of $g_{Y}$, which is essential for the unification, is best explained in the framework of GUT. It seems to imply the real presence of GUT at the $10^{16} \mathrm{GeV}$ scale.

A realization of the GUT idea, however, would be quite complicated in the 4 dimensional (4D) spacetime: Even spontaneous symmetry breaking mechanism of GUT into the standard model (SM) gauge symmetry $G_{S M}$ without fine-tuning and without leaving unwanted pseudo-Goldstones and triplet Higgs is not simple. Accordingly, even the gauge coupling unification consistent with the low energy value of $\sin ^{2} \theta_{W}(\approx 0.23)$ in GUT, which provided a strong evidence supporting GUT, is non-trivial.

In higher dimensional spacetime, however, SUSY GUT idea can be simply realized, if the compactification mechanism of the extra dimensions is associated with GUT and SUSY breakings. For instance, in 5D orbifold compactification with $S^{1} /\left(Z_{2} \times Z_{2}^{\prime}\right)$ [1], the boundary conditions consistent with $Z_{2}$ parities lead to $\mathscr{N}=2$ SUSY breaking (in terms of 4D SUSY) into $\mathscr{N}=1$. The other $Z_{2}^{\prime}$, which is originated from the translational symmetry of $S^{1}$, can be optionally utilized to break the gauge symmetry $G_{\mathrm{GUT}}$. As a result, below the compactification scale $(\approx 1 / R)$ we have $\mathscr{N}=1$ MSSM, while above the $1 / R$ scale the original $G_{\mathrm{GUT}}$ as well as $\mathscr{N}=2$ SUSY is effectively restored, because of the presence of KaluzaKlein (KK) modes. Unlike in the 4D GUTs, the notorious doublet/triplet splitting problem is also very easily resolved (just by boundary conditions). Thus, this model maintains the nice features of the GUT idea in a simple way. Unfortunately, however, 5D orbifold SUSY GUT is not renormalizable and so quantum mechanically not predictable. It needs to be supported by the fundamental theory such as the string theory.

Nonprime Orbifold. By employing the nonprime orbifold compactification of the heterotic string theory, we can obtain 5D or 6D orbifold SUSY GUTs. In the large limit of the extra 2D (1D), a 4D theory would become an effective 6D (5D) string theory with $\mathscr{N}=2$ or 4 SUSY. In the orbifold compactification, the $\mathscr{N}=2$ SUSY requires the presence of a sub-lattice invariant under a given twist action, i.e. a torus. Hence, there is no twisting for strings on it. Background moduli such as radius (or metric) can be encoded only in the zero modes' momenta of untwisted bosonic strings [2], which are interpreted as KK modes in the 4D spacetime. Since twisted strings are stuck to orbifold fixed points, they cannot accommodate moduli. In order to introduce a modulus such as arbitrarily large radius and to discuss the relevant KK excitations, therefore, we need to employ an orbifold compactification providing an invariant sub-lattice.

In nonprime orbifold compactifications, some higher twist sectors turn out to behave like in the invariant sublattice preserving $\mathscr{N}=2$ SUSY. KK excited states can arise from such higher twist sectors in nonprime orbifold compactifications [2]. Moreover, orbifold string compactifications must break a gauge symmetry as well as SUSY, because of the modular invariance of the string theory. Accordingly, the gauge symmetry could be enhanced by including $\mathrm{KK}$ massive states above the $1 / R$ scale. Hence, it is possible to construct a higher dimensional SUSY GUT with the help of KK states [4, 5, 6]. We are particularly interested in the nonprime orbifold $\mathbf{Z}_{12-I}$ which has some interesting features [3, 4, 5]. This method can be easily extended to the other nonprime orbifold compactifications.

The $\mathbf{Z}_{12-I}$ orbifold is defined with the twist vector 
$\phi=\left(\frac{5}{12}, \frac{4}{12}, \frac{1}{12}\right)$, acting on the complexified three dimensional string: $X_{L, R}^{A}(2 \pi)=e^{+2 \pi i \phi_{A}} X_{L, R}^{A}(0), A=1,2,3$. Similarly, $X_{L, R}^{A *}$ acquires $e^{-2 \pi i \phi_{A}}$. This orbifold can be the $\mathrm{SO}(8) \times \mathrm{SU}(3)$ or $\mathrm{F}_{4} \times \mathrm{SU}(3)$ lattice, where the $\mathrm{SU}(3)$ lattice corresponds to the second complex plane. We will assume that the size $R$ of the second torus is relatively larger than the sizes of the first and the second tori.

The modular invariance of the orbifolded string theory requires to consider all the possible sectors with the boundary conditions, $0 \times \phi, 1 \times \phi, 2 \times \phi, \cdots, 11 \times \phi$, which are called " $U$ ", " $T_{1}, "$ " $T_{2}, \cdots, \cdots, T_{11}$ " sectors, respectively. The sector of $12 \times \phi$ is identified with the $U$ sector. In the $T_{3}, T_{6}$, and $T_{9}$ sectors of $\mathbf{Z}_{12-I}$, where the boundary conditions are given by $3 \phi, 6 \phi$, and $9 \phi$, respectively, the second (complexified) sub-lattice remains untwisted. Namely, the second sub-lattice is just an ordinary torus in the $T_{3}, T_{6}$, and $T_{9}$ sectors. The KK massive states can arise only from the sectors associated with invariant sub-lattices, and they indeed respect $\mathscr{N}=2$ SUSY [2, 3].

Partition Function. Many important physical information such as the mass-shell condition, GSO projection, and so on, can be extracted from the one loop partition function. The one-loop amplitude by closed strings has the topology of a torus. A world-sheet torus can be parametrized by $\sigma_{1}+\tau \sigma_{2}\left(0 \leq \sigma_{1}, \sigma_{2} \leq 2 \pi\right)$, where $\tau$ $\left(\equiv \tau_{1}+i \tau_{2}\right)$ is the modular parameter. On the world-sheet torus, we have two boundary conditions, $\left[g^{k}, g^{l}\right]$ (or simply $[k, l])$, where $g^{k}\left(g^{l}\right)$ implies the action of the order $k(l)$ twisted string boundary conditions in the $\sigma_{1}\left(\sigma_{2}\right)$ direction. In the $[k, l]$ sectors with $k$ and $l=0,3,6,9$, $X_{L, R}^{i}$ in the longitudinal directions of the second complex plane $(i=3,4)$ are untwisted, and so KK massive states can arise from there. On the other hand, in the $[k, l]$ sectors with $k$ or $l \neq 0,3,6,9$, where only $\mathscr{N}=1$ SUSY is preserved, there do not appear KK excited states.

The full partition function, which is given by the summation of the $[k, l]$ sectors' contributions, should be invariant under the modular transformations, i.e. "S" $(\tau \rightarrow$ $-1 / \tau)$ and "T" $(\tau \rightarrow \tau+1)$ transformations. Under the $\mathrm{S}(\mathrm{T})$ transformation, a $[k, l]$ sector is cast to the $[l,-k]$ $([k, l+k])$ sector. For $\tau \rightarrow-1 / \tau$ and $\tau \rightarrow \tau+1$, therefore, the $[k, l]$ sectors with $k, l=0,3,6,9$ are interchanged each other only inside $\{[k, l]$ sectors $; k, l=0,3,6,9\}$, decoupled from the other sectors with $k, l \neq 0,3,6,9$. The partition function invariant under the $\mathrm{S}$ and $\mathrm{T}$ transformations in $U, T_{3}, T_{6}, T_{9}$ takes typically the following form [3]:

$$
\begin{gathered}
\frac{1}{\lambda^{2}}\left[\sum_{\vec{\mu} \in \Lambda_{2}^{*}, \vec{\zeta} \in \Lambda_{2}} \frac{q^{(\vec{L}+\vec{\zeta})^{2} / 2}}{[\eta(\tau)]^{2}} \frac{\bar{q}^{(\vec{L}-\vec{\zeta})^{2} / 2}}{[\bar{\eta}(\bar{\tau})]^{2}}\right] \times \\
{\left[\sum_{P \in \Lambda_{16}, s \in \Lambda_{8}} \frac{q^{\left(L^{I}\right)^{2} / 2}}{[\eta(\tau)]^{16}} \frac{\bar{q}^{(\tilde{L})^{2} / 2}}{[\bar{\eta}(\bar{\tau})]^{4}} \times e^{2 \pi i l \Theta_{k}}\right]\left|\widehat{Z}_{[k, l]}^{X}\right|^{2} .}
\end{gathered}
$$

which is of order $1 / R^{2}\left(\ll 1 / \alpha^{\prime}\right)$ because of the inverse metric of SU(3) lattice, $g^{a b}$. For massless states in the $U$, $T_{3}, T_{6}, T_{9}$ sectors, $P^{I} W_{b}^{I}=$ integer should be guaranteed.

Both massless and KK massive states should satisfy

$$
\frac{\left(L^{I}\right)^{2}}{2}+\sum_{i=j, \bar{j}} N_{i}^{L} \tilde{\phi}_{i}-1+\frac{c_{k}}{2}=\frac{(\tilde{L})^{2}}{2}-\frac{1}{2}+\frac{c_{k}}{2}=0,
$$


where $i$ runs over $\{1,2,3, \overline{1}, \overline{2}, \overline{3}\}$, and $\tilde{\phi}_{j} \equiv k \phi_{j} \bmod \mathrm{Z}$ such that $0<\tilde{\phi}_{j} \leq 1$, and $\tilde{\phi}_{\bar{j}} \equiv-k \phi_{j} \bmod Z$ such that $0<$ $\tilde{\phi}_{\bar{j}} \leq 1 . N_{i}^{L}$ indicates the oscillator numbers $(0,1,2, \cdots)$. For $c_{k}$ in the world sheet vacuum energy, see Ref. [3, 4].

The generalized GSO projector is read from the coefficient of $q^{\alpha^{\prime} M_{L}^{2} / 4} \bar{q}^{\alpha^{\prime} M_{R}^{2} / 4}$ in the partition function:

$$
\mathscr{P}_{k}=\frac{1}{N} \sum_{l} \tilde{\chi}\left(\theta^{k}, \theta^{l}\right) e^{2 \pi i l \widetilde{\Theta}_{k}}
$$

where the complete form of $\widetilde{\Theta}_{k}$ and the degeneracy factor $\tilde{\chi}\left(\theta^{k}, \theta^{l}\right)$ are presented in Ref. [3]. $N=12$ for the massless states. However, $N=4$ for the KK massive states, because KK massive states appear only in the $U, T_{3}, T_{6}$, and $T_{9}$ sectors. Hence, $l$ in Eq. (9) runs 0,3,6,9 for KK states, whereas $l=0,1,2, \cdots, 11$ for massless states.

Threshold Correction. The general expression for the moduli dependent stringy threshold correction to the gauge couplings $\Delta_{i}$ can be obtained as [2]

$$
\Delta_{i}=\frac{\left|G^{\prime}\right|}{|G|} \cdot b_{i}^{N=2} \int_{\Gamma} \frac{d^{2} \tau}{\tau_{2}}\left(\hat{Z}_{\text {torus }}(\tau, \bar{\tau})-1\right),
$$

where $b_{i}^{N=2}$ denotes the beta function coefficient of $\mathscr{N}=$ 2 SUSY sector by the KK modes. Since $G=\mathbf{Z}_{4} \times \mathbf{Z}_{3}$ and $G^{\prime}=\mathbf{Z}_{3}$ in our case, $\frac{\left|G^{\prime}\right|}{|G|}=\frac{1}{4}$. The $\hat{Z}_{\text {torus }}(\tau, \bar{\tau})(\equiv$ $\left.\sum_{\vec{P}_{L}, \vec{P}_{R}} q^{P_{L}^{2} / 2} \bar{q}^{2} / 2\right)$ is given by

$$
\hat{Z}_{\text {torus }}(\tau, \bar{\tau})=\sum_{\vec{\mu}, \vec{\zeta}} \frac{1}{\lambda^{2}} q^{(\vec{L}+\vec{\zeta})^{2} / 2} \bar{q}^{(\vec{L}-\vec{\zeta})^{2} / 2},
$$

where $\vec{L}$ is given in Eq. (2).

Thus, if a gauge group $G$ is broken to $\tilde{H}$ by the Wilson lines and further broken to $H$ by orbifolding, the renormalized gauge couplings of $H$ at low energies is

$$
\begin{aligned}
\frac{16 \pi^{2}}{g_{H}^{2}(\mu)}= & \frac{16 \pi^{2}}{g_{*}^{2}}+b_{H}^{0} \log \frac{M_{*}^{2}}{\mu^{2}}-\frac{b_{\tilde{H}}}{4}\left[\log \frac{R^{2}}{\alpha^{\prime}}+1.89\right] \\
& +\frac{b_{\tilde{H}+G / \tilde{H}}}{4}\left[\frac{2 \pi R^{2}}{\sqrt{3} \alpha^{\prime}}-0.30\right] .
\end{aligned}
$$

$b_{H}^{0}$ is the beta function coefficient contributed by $\mathscr{N}=1$ SUSY sector states projected by $P^{I} W^{I}=$ integer. $b_{\tilde{H}}$ $\left(b_{G / \tilde{H}}\right)$ is by the $\mathscr{N}=2$ SUSY sector states projected by $P^{I} W^{I}=$ integer $\left(P^{I} W^{I} \neq\right.$ integer $)$.

The $R^{2}$ coefficient is contributed by all the states charged under $G$. Hence, the difference $g_{H i}{ }^{-2}-g_{H j}{ }^{-2}$ does not get an $R^{2}$ dependent piece if the gauge groups are unified to $G$ above the $1 / R$. However, the logarithmic contribution is still present. The absolute values of the constant and the (quadratically divergent) $R^{2}$ term are reliable since their calculations are based on the fundamental theory.
Model/Conclusions. Let us propose a string model for MSSM [5]. It turns out to be embedded in a 6D SU(8) GUT above the $1 / R\left(\sim M_{\mathrm{GUT}}\right)$ scale [3]. We take the following forms of a shift vector and a Wilson line, which are associated with the boundary conditions of $X_{L}^{I}$ :

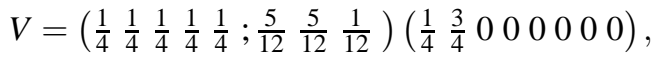

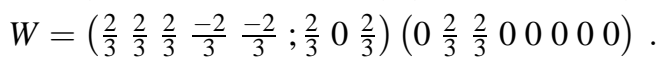

They satisfy Eqs. (5)-(7). The massless states in gauge sector are given by the $\mathrm{E}_{8} \times \mathrm{E}_{8}^{\prime}$ root vectors $\left(P^{2}=2\right)$ satisfying $P \cdot V=$ integer and $P \cdot W=$ integer with $s \cdot \phi=0$. They are $\left(1-10 ; 00 ; 0^{3}\right)\left(0^{8}\right)^{\prime}$, $\left.\left(000 ; \underline{1-1} ; 0^{3}\right)\left(0^{8}\right)^{\prime}, \overline{\left(0^{8}\right)(00} ; \pm 1 \pm 1000\right)^{\prime}$, where the underlined entries allow all possible commutations. Thus, the resulting gauge group is

$$
\left[\left\{\mathrm{SU}(3)_{\mathrm{c}} \times \mathrm{SU}(2)_{\mathrm{L}} \times \mathrm{U}(1)_{\mathrm{Y}}\right\} \times \mathrm{U}(1)^{4}\right] \times\left[\mathrm{SO}(10) \times \mathrm{U}(1)^{3}\right]^{\prime} .
$$

The hypercharge of $\mathrm{U}(1)_{\mathrm{Y}}$ is defined with the standard one, $Y=\sqrt{\frac{3}{5}}\left(\frac{1}{3} \frac{1}{3} \frac{1}{3} \frac{-1}{2} \frac{-1}{2} ; 0^{3}\right)\left(0^{8}\right)^{\prime}$. Here the normalization factor $\sqrt{3 / 5}$, which leads to $\sin ^{2} \theta_{W}=\frac{3}{8}$ at the string scale, is determined by the current algebra in the heterotic string theory. The important features of this model are summarized as follows:

- One family of the MSSM matter comes from the $U$ sectors and two families from $T_{4}$. They all compose full sets of $\mathrm{SO}(10)$ spinor representations. The other matter form vector-like representations under $G_{S M}$.

- The MSSM two Higgs doublets $\left(000 \pm 10 ; 0^{3}\right)\left(0^{8}\right)^{\prime}$ are from the $U$ sector, whereas the triplets $\left( \pm 10000 ; 0^{3}\right)\left(0^{8}\right)^{\prime}$ are absent there.

- The exact matter parity can be defined on a vacuum, where all extra U(1)s are broken and all extra states achieve superheavy masses by SM singlet VEVs.

- Above the $1 / R$ scale $\left(\sim M_{\mathrm{GUT}}\right)$, the model becomes a 6 D SU(8) SUSY GUT, and the MSSM gauge couplings are unified around $10^{17} \mathrm{GeV}$. The two Higgs doublets are included in the 6D gauge multiplet.

\section{REFERENCES}

1. Y. Kawamura, Prog. Theor. Phys. 105, 999 (2001); L. J. Hall and Y. Nomura, Phys. Rev. D65, 125012 (2002). B. Kyae, C. A. Lee and Q. Shafi, Nucl. Phys. B683, 105 (2004).

2. L. J. Dixon, V. Kaplunovsky and J. Louis, Nucl. Phys. B355, 649 (1991); K. S. Narain, M. H. Sarmadi and E. Witten, Nucl. Phys. B279, 369 (1987).

3. J. E. Kim and B. Kyae, Phys. Rev. D 77, 106008 (2008).

4. J. E. Kim and B. Kyae, Nucl. Phys. B770, 47 (2007).

5. J. E. Kim, J.-H. Kim and B. Kyae, JHEP 0706, 034 (2007). See also J. E. Kim, J. H. Kim, and B. Kyae, arXiv:0710.4868 [hep-th].

6. O. Lebedev et al., Phys. Rev. D 77, 046013 (2008). 